\title{
Neurobiological and clinical effect of metacognitive interpersonal therapy vs structured clinical model: study protocol for a randomized controlled trial
}

\author{
Laura Rosa Magni ${ }^{1}$, Antonino Carcione ${ }^{2,3}$, Clarissa Ferrari ${ }^{4}$, Antonio Semerari ${ }^{2,3}$, llaria Riccardi ${ }^{2,3}$, Giuseppe Nicolo ${ }^{\text {2,3,5, }}$, \\ Mariangela Lanfredi ${ }^{1}$, Laura Pedrini ${ }^{1}$, Maria Cotelli ${ }^{6}$, Luisella Bocchio ${ }^{7}$, Michela Pievani ${ }^{8}$, Roberto Gasparotti ${ }^{9}$, \\ Roberta Rossi ${ }^{1 *}$ and CLIMAMITHE Study group
}

\begin{abstract}
Background: Borderline Personality Disorder (BPD) is a complex and debilitating disorder, characterized by deficits in metacognition and emotion dysregulation. The "gold standard" treatment for this disorder is psychotherapy with pharmacotherapy as an adjunctive treatment to target state symptoms.

The present randomized clinical trial aims to assess the clinical and neurobiological changes following Metacognitive Interpersonal Therapy (MIT) compared with Structured Clinical Management (SCM) derived from specific recommendations in APA (American Psychiatric Association) guidelines for BPD.

Methods: The study design is a randomized parallel controlled clinical trial and will include 80 BPD outpatients, aged 18-45 enrolled at 2 recruitment centers. Primary outcome will be the clinical change in emotion regulation capacities assessed with the Difficulties in Emotion Regulation Scale (DERS). We will also investigated the effect of psychotherapy on metacognitive abilities and several clinical features such as BPD symptomatology, general psychopathology, depression, personal functioning, and trait dimensions (anger, impulsivity, alexithymia). We will evaluate changes in brain connectivity patterns and during the view of emotional pictures. A multidimensional assessment will be performed at the baseline, at 6, 12, 18 months. We will obtain structural and functional Magnetic Resonance Images (MRIs) in MIT-Treated BPD $(N=30)$ and SCM-treated BPD $(N=30)$ at baseline and after treatment, as well as in a group of 30 healthy and unrelated volunteers that will be scanned once for comparison.

Discussion: The present study could contribute to elucidate the neurobiological mechanisms underlying psychotherapy efficacy. The inclusion of a multidisciplinary study protocol will allow to study BPD considering different features that can affect the treatment response and their reciprocal relationships.

Trial registration: NCT02370316. Registered 02/24/2015.

Keywords: Borderline personality disorder, Psychotherapy, Neuroimaging, Metacognition, Emotion dysregulation
\end{abstract}

\footnotetext{
* Correspondence: rrossi@fatebenefratelli.eu

${ }^{1}$ Unit of Psychiatry, IRCCS Istituto Centro San Giovanni di Dio Fatebenefratelli,

Brescia, Italy

Full list of author information is available at the end of the article
}

(c) The Author(s). 2019 Open Access This article is distributed under the terms of the Creative Commons Attribution 4.0 International License (http://creativecommons.org/licenses/by/4.0/), which permits unrestricted use, distribution, and reproduction in any medium, provided you give appropriate credit to the original author(s) and the source, provide a link to the Creative Commons license, and indicate if changes were made. The Creative Commons Public Domain Dedication waiver (http://creativecommons.org/publicdomain/zero/1.0/) applies to the data made available in this article, unless otherwise stated. 


\section{Background}

Borderline Personality Disorder (BPD) is one of the most common disorders in clinical settings. Psychotherapy is the primary treatment for BPD, with pharmacotherapy as an adjunctive treatment to target state symptoms [1]. In the last two decades, numerous psychotherapeutic approaches have been proposed for this diagnosis and their effectiveness in reducing symptoms and dysfunctions in behaviors seemed to be well supported, even though data on the improvement in social functioning is less optimistic [2-4]. Furthermore, these specialist treatments seem to have similar effects despite distinct theories and interventions. Moreover, despite progress, how psychological therapies produce this improvement is not fully understood [5]. Neurobiological studies could clarify the mechanism of change of psychotherapy for BPD and this could improve our knowledge of the pathophysiology underlying the disease. In particular, several MRI studies explored the neurobiological correlates of the disorder, showed volume reduction in amygdala and hippocampus [6-9], thickness decrease of the prefrontal cortex [10-12], and volume reduction in various regions of the temporal and parietal lobes [13, 14], as compared with healthy subjects. In the field of fMRI studies, the hyperactivity of the amygdala and hypo-activation of frontal areas in response to emotional stimuli in BPD samples [15] seems to be one of the most robust finding. These results were interpreted as the biological substrate of the core symptoms of the disease and, in particular, emotional dysregulation. The key question is whether psychotherapy is able to impact cerebral structures and functional activities and connectivity.

While the majority of the neuroimaging studies of psychotherapeutic treatments have been conducted on Axis I disorders, such as obsessive-compulsive disorder [15-18], mood disorders [19-21], panic disorder [22], social anxiety disorder [23], specific phobia $[24,25]$, and posttraumatic stress disorder (PTSD) [26], only a few studies explored the impact of psychotherapy in personality disorder and the most are in the context of Dialectical Behavioral Therapy for BPD. Goodman and colleagues showed BPD patients showed an overall decrease in amygdala after 12 months of DBT while the overall amygdala activation of the $\mathrm{HC}$ (healthy control) was comparable at baseline and 1 year follow-up [27].

A previous pilot study on 6 BPD patients showed comparable results, in particular a decreasing hemodynamic response to negative stimuli in the right-sided anterior cingulate, temporal and posterior cingulate cortices as well as in the left insula after a 12-week in-patient treatment program [28]. In another interesting study on a 12-weeks DBT program, patients exhibited reduced activity and increased connectivity in neural networks related to salience processing and emotion regulation after treatment $[29,30]$. Preliminarily, some effects on brain structure, in terms of increased gray matter volumes in regions that are critically implicated in emotion regulation and higher-order functions, such as mentalizing, have been described [31].

Furthermore, Perez and colleagues [32] showed that after 1 year of Transference-Focused Psychotherapy [4] BPD patients showed relatively increased activation in dorsal prefrontal (dorsal anterior cingulate, dorsolateral prefrontal, and frontopolar cortices) in relation with treatment effect, and relatively decreased activation in ventrolateral prefrontal cortex and hippocampal after the intervention. Noteworthy, an increased left dorsal anterior cingulate cortex activation resulted positevely correlate to clinical improvement in constraint, while left posterior-medial orbitofrontal cortex/ventral striatum activation and negatively with right amygdala/parahippocampal activation seemed to be positively associated to clinical improvement in affective lability correlated. These results are very intriguing and they gave a great contribution to clarify possible mechanisms associated to clinical changes induced by the psychotherapy. No neuroimaging study has yet assessed the effect of other psychotherapy interventions on neurobiological features, e.g. approaches specifically oriented to increase mentalizing [3] or metacognition [33] that are often compromised in BPD and represent one of the core features in BPD patients. Metacognition, as conceptualized by Semerari [33], is the general capacity to think about thinking. Scarce metacognitive abilities have been associated to the difficulty to deal with interpersonal problems and deficit in using problem-solving strategies and choosing adaptive behaviors [34]. Metacognitive Interpersonal Therapy (MIT) is a cognitive behavior-based psychotherapeutic approach aimed to increase metacognitive abilities in order to improve general personality functioning and to promote better interpersonal relationships [35]. More in detail, MIT is designed to support patients in learning to recognize and integrate different mental states and in improving their ability to solve interpersonal problems using mentalistic knowledge of themselves and others.

The aim of the present study is twofold. The primary outcome will be the change in emotion dysregulation, measured by the Difficulties in Emotion Regulation Scale - DERS, [36, 37] after 12 months of MIT treatment in subjects with BPD. The secondary outcomes will be the effect of MIT on neurobiological (changes in cerebral patterns of activation in response to emotional visual stimuli during fMRI scans) and other clinical features. Lastly, we will study the correlation between patients' metacognitive profiles and structural and functional brain imaging features. 


\section{Method/design}

\section{Trial design}

The study design is a randomized parallel controlled clinical trial. For an overview of the proposed flow of participants, see Fig. 1. The present study protocol was written in accordance with the Standard Protocol Items: Recommendations for Interventional Trials (SPIRIT) [38]; copies of the SPIRIT Checklist and figure have been included in Table 1 and Additional file 1.

\section{Participants and study setting}

Eighty BPD outpatients will be enrolled at 2 recruitment centres (IRCCS Istituto Centro San Giovanni di Dio, Brescia, Italy; Third Center of Cognitive Psychotherapy, Rome, Italy Scuola Italiana di Cognitivismo Clinico-SICC, Rome). A group of 30 healthy volunteers will be enrolled as reference group IRCCS Istituto Centro San Giovanni di Dio, Brescia.

\section{Inclusion and exclusion criteria}

The inclusion criteria for patients will be: age 18-45 and a diagnosis of BPD (DSM-IV-TR); informed consent.
Patients will be excluded whether a lifetime diagnosis of schizophrenia, schizoaffective disorder, substance abuse or dependence in the 3 months before the enrolment, bipolar disorder, organic mental syndromes, dementia or cognitive impairment, relevant neurological signs will be ascertained. Furthermore, we will exclude pregnant women or lactating or patients receiving concurrent psychotherapy.

HCs will be represented by healthy volunteers without any cognitive impairment or psychiatric/neurologic condition, including alcohol/substance abuse.

\section{Clinical assessment}

Independent expert psychologists will conduct the clinical assessment. Clinical evaluation are scheduled at the baseline, after 6, 12 (end of treatment), and 18 months.

The Structured Clinical Interview for DSM disorder $[39,40]$ will be used to define the diagnosis and collect data on comorbidities.

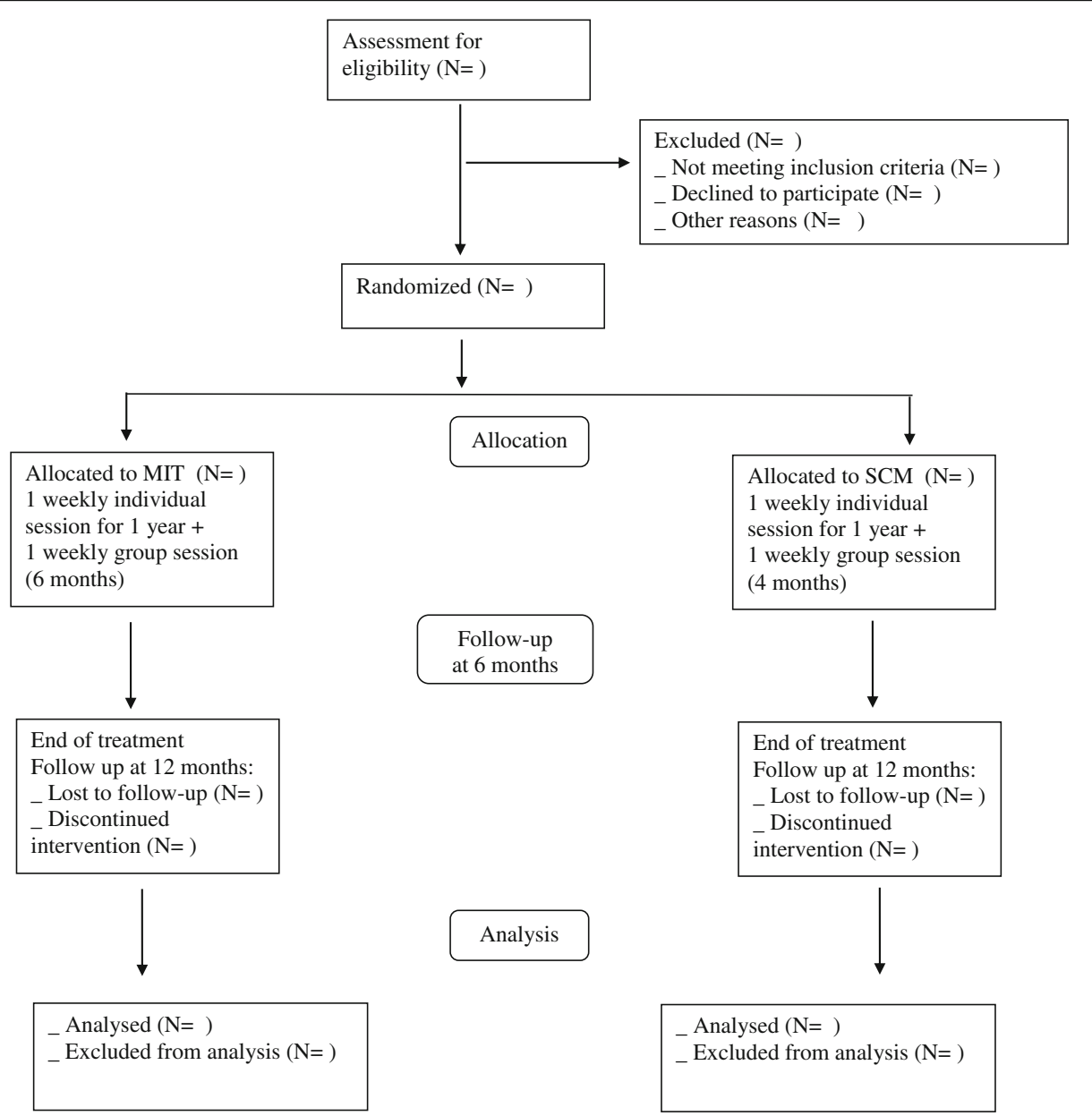

Fig. 1 Study Flowchart. MIT: Metacognitive Interpersonal Therapy; SCM Structured Clinical Management 
Table 1 SPIRIT 2013 Figure for CLIMAMITHE study

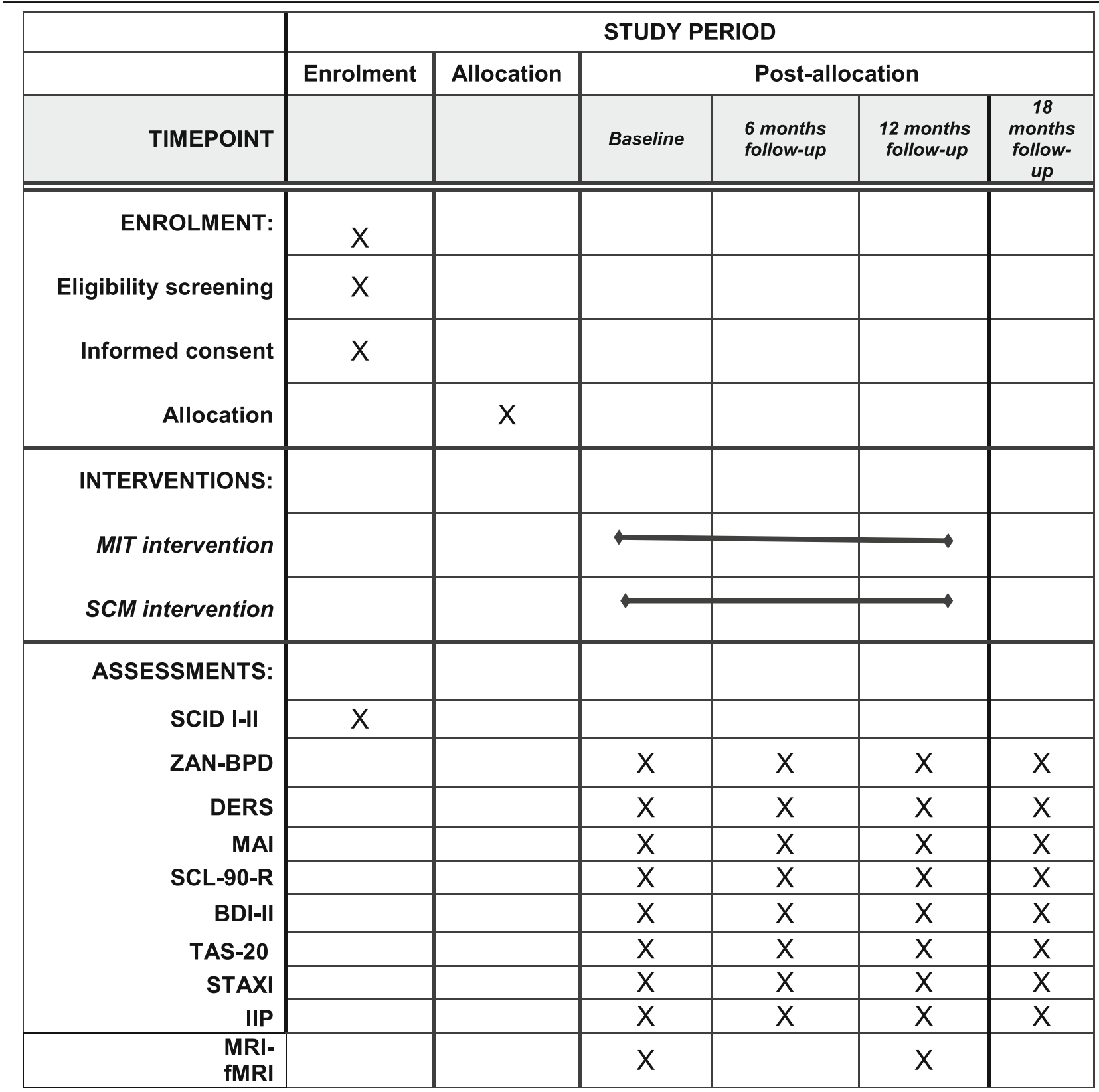

MIT Metacognitive Interpersonal Therapy, SCM Structured Clinical Management, SCID I-II structured Clinical Interview for DSM disorder, axis I and axis II, ZAN-BPD Zanarini Rating Scale for Borderline Personality Disorder, DERS Difficulties in Emotion Regulation Scale, MAI Metacognitive Assessment Interview, SCL-90-R Symptoms Checklist-90- Revised, BDI-II Beck Depression Inventory. TAS-20 Toronto Alexithymia Scale, STAXI State and Trait Anger Expression Inventory, IIP Inventory of Interpersonal Problems, MRI-fMRI Magnetic Resonance Imaging- Functional Magnetic Resonance Imaging

\section{Interventions}

Patients will be randomly allocated to one of two interventions: Metacognitive Interpersonal Treatment (MIT) [35] or Structured Clinical Management (SCM) [1] delivered in each recruitment centre.

MIT is a cognitive behavioral-oriented psychotherapy designed to ameliorate metacognitive abilities and to enhance interpersonal relationships [35, 41, 42]. Metacognition pertain to a comprehensive set of cognitive and affective skills aimed to identify mental states, reasoning about them, and attributing them to themselves and others [33, 43]. These skills allow people to understand the reason why other persons react in such way, on the basis of own regularities and constructing personal meaning over their lifespan [44]. MIT is conceptualized to target the general psychopathology of personality. Treatment consists in 50-min one weekly individual session and a metacognitive skill training group (90 min) that 
cover a period of about 6 months during the year of the treatment. The MIT is manualized and follows a goal hierarchy, as proposed by the authors of the theoretical model [35]. In particular, the main goals of the treatment are: 1) the assessment of symptoms, mental state, metacognitive functions, interpersonal problems, emotion regulation skills; 2) intervention on the aspects that interfere with the therapeutic alliance and/or threaten the patient's life (in particular, self-harming/ suicidal behaviors); 3) intervention on symptoms that cause suffering to the patient; 4) intervention to promote the integration of different mental states MIT sessions will be supervised by the authors of the theoretical model through audio-record and the analyses of the narratives.

SCM is an evidence-based intervention that reflects "best general psychiatric treatment for BPD" and it's feasible for use by "generalist mental health clinicians" with minimal additional training [45]. It was developed based on "expert consensus" about what general practices work best for treating this condition. SCM is the active comparator used in several study on BPD psychotherapy showing to be effective across an array of clinical outcomes [46]. SCM is tailored on BPD symptomatology and employs a supportive approach with case management and advocacy support. There is an emphasis on psychoeducation, problem-solving, explicit safety planning, medication review and assertive follow-up if appointments are missed. As the comparator, SCM treatment consists in 50-min one weekly individual session and a problem solving group (90 min) that cover a period of about 6 months during the year of the treatment.

Treatment retention was measured by the total number of weeks with at least one session and the number of weeks from the first to the last session attended. Patients were classified as having completed treatment if the time between the first and last sessions was at least 12 months between the 1st and the last session. Dropouts will be defined as those missing four consecutive sessions with no ascertained reason.

Treatment adherence. MIT therapists will be supervised throughout the duration of treatments, and treatment fidelity will be assessed using audiotaped sessions. For each therapist, 2 entire sessions will be recorded and evaluated by 2 senior therapists who developed the MIT, to ensure that the treatment is being done in a rigorous, reproducible, and similar way. As part of the assessment of fidelity, a treatment manual will be developed (as describe above). After each session, each MIT therapist will be required to fulfill a checklist on MIT strategies and techniques in order to monitor the fidelity. For SCM, bimonthly supervision will be mandatory to monitor the case management.

\section{Therapists' requirements}

Psychotherapies will be delivered by 12 "expert therapists", with 4-year training in psychotherapy (psychodynamic- or CBT-oriented), a minimum of 2 years of clinical experience and a minimum of 1 year of experience treating BPD patients. More specifically, MIT therapists involved in the project will have received an intensive training in MIT (48 h), 4 years with Cognitive Behavioral are required. For SCM therapists, a $16 \mathrm{~h}$-training on SCM will be delivered, no specific theoretical model will be required but expertise in treating BPD.

In each arm, patients will be randomly assigned to therapists within 2 weeks from the randomization.

\section{Pharmacological treatment}

In order to reduce the possible confounding effect of pharmacotherapy, both on clinical and neuroimaging measures, an adaptation of the APA Guidelines [1] will be adopted to harmonize the prescriptions. Briefly, it includes the following principles: 1) Treatment is symptom specific, directed at particular behavioral dimensions; 2) Affective dysregulation and impulsivity/ aggression are risk factors for suicidal behavior, self-injury, and are given high priority in selecting pharmacological agents; 3) Medication targets both acute symptoms (e.g., anger treated with dopamine-blocking agents) and chronic vulnerabilities (e.g., temperamental impulsivity treated with serotonergic agents).

Symptoms to be targeted are divided in three groups: Affective dysregulation symptoms, Impulsive behavioral symptoms and Cognitive-perceptual symptoms and each of this has specific recommendation.

\section{Primary outcome}

The primary outcome measure will be changes in emotion regulation, measured by the Difficulties in Emotion Regulation Scale (DERS) [36, 37]. This scale is a 36-item self-report questionnaire comprising a total score and six dimensions: (1) Non-acceptance of emotional responses (6 items); (2) Difficulties engaging in goal-directed behavior (5 items); (3) Impulse control difficulties (6 items); (4) Lack of emotional awareness (6 items); (5) Limited access to emotion regulation strategies (8 items); (6) Lack of emotional clarity ( 5 items). The scale showed good psychometric properties, in terms of internal consistency (Cronbach's alpha between 0.80 and 0.93 ), construct and predictive validity and adequate test-retest reliability.

\section{Secondary outcomes}

A multidimensional evaluation with standardized tools will assess secondary outcomes in several psychological domains.

Metacognition Assessment Interview (MAI) [33, 47, 48]. Metacognitive functions will be measured with the MAI, a 
semi-structured clinical interview aimed to evaluate the metacognitive abilities of patients. The interviewers ask for a report of an emotionally meaningful experience or life event occurred in the previous 6 months, in which the subject and another person was involved. After the spontaneous report of the situation, interviewers asked a series of specific questions designed to evaluate the four metacognitive subfunctions (monitoring, integration, differentiation, and decentration).

Symptoms Check-list 90 Revised (SCL-90-R) [49]. General psychopathology will be assessed with the SCL-90-R, a 90 item self-report inventory aimed to measure the presence of psychological symptoms. The SCL-90-R assessed nine symptom dimensions: (1) Somatization; (2) Obsessive-Compulsive; (3) Interpersonal Sensitivity; (4) Depression; (5) Anxiety; (6) Hostility; (7) Phobic Anxiety; (8) Paranoid Ideation; and 9) Psychoticism. For the purpose of our study, we will used the Global Severity Index (GSI), which is the mean value of all of the items, and is considered a measure of global symptomatic distress.

Beck Depression Inventory II (BDI-II) [50]. Depressive symptoms will be evaluated by the BDI-II, a 21-item self-report questionnaire measuring the severity of depressive symptoms in the past 2 weeks with higher scores correlating higher levels of depression.

State-Trait Anger Expression Inventory (STAXI) [51]. State and Trait anger will be assessed with STAXI-2, that is a 57 -item inventory which measures the intensity of anger as an emotional state (State Anger) and the disposition to experience angry feelings as a personality trait (Trait Anger). The instrument consists of six scales measuring the intensity of anger and the disposition to experience angry feelings. Items consist of 4-point scales that assess intensity of anger at a particular moment and the frequency of anger experience, expression, and control.

Barratt Impulsiveness Scale (BIS) [52] is a questionnaire aimed to evaluated impulsiveness. The BIS-11 identifies three factors that express three different dimensions of impulsivity: Motor impulsiveness, Impulsivity without planning and Cognitive impulsivity and provides a general index of the construct, as a total score. These scales have shown a good reliability and validity with other measures of the same dimension and the total score of the BIS-11 is an internally consistent measure of impulsiveness.

Interpersonal functioning will be assessed by Inventory of Interpersonal Problems (IIP) [53]. IIP is a 57 items self-report, evaluating different dimensions of interpersonal features (interpersonal sensitivity, interpersonal ambivalence, aggression, need for social approval, and lack of sociability).

Alexithymia will be measured by Toronto Alexithymia Scale (TAS-20) [54], a self-report consisting of 20 items rated on a 5-point Likert scale. TAS-20 provide a total score and three subscales: Difficulties Identifying Feelings;
Difficulties Describing Feelings; and Externally Oriented Thinking, which refers to a specific tendency to focus on superficial matters and to avoid emotional thinking (Bagby, et al., 1994).

Childhood traumatic experiences will be evaluated by the Childhood Trauma Questionnaire (CTQ) [55]. The CTQ includes a 28 -items that measures 5 types of maltreatment - emotional, physical, and sexual abuse, and emotional and physical neglect.

The attachment experience will be assessed by the Attachment Style Questionnaire (ASQ) [56] that is a 40-item survey that uses a 6-point Likert-type scale. The ASQ yields five factor scores: one is a factor representing secure attachment, the other four represent a particular aspect of insecure attachment.

Data on demographics, suicide attempts, self-injury and aggression episodes, hospitalizations, and pharmacotherapy will be collected.

At the baseline, the neuropsychological test battery included measures used to assess nonverbal reasoning (Raven's Colored Progressive Matrices), verbal fluency (phonemic and semantic), visuospatial capacity (Rey-Osterrieth Complex Figure Copy), and attention and executive function (Trail Making Test, Wisconsin Card Sorting Test, Stroop Test), memory (Story Recall, Rey-Osterrieth Complex Figure Recall, Digit Span). All of the neuropsychological tests were administered and scored according to standard procedures [57].

Furthermore, all participants completed the Interpersonal Reactivity Index (IRI) [58], the Reading the Mind in the Eyes (RME) [59], the Facially expressed emotion labeling (FEEL) [60] and an experimental task to assess emotional priming (Emotional Priming Paradigm, EPP) [61].

Additionally, we will collect blood samples at baseline and at the different time of observation in order to explore possible peripheral biomarkers of emotional dysregulation and related to the limbic system, neuroplasticity, presence of inflammation and stress response and to observe modifications during treatment.

\section{Neuroimaging protocol}

Structural, functional and diffusion MRI following the ADNI protocol [62] will be acquired in BPD patients twice, at the baseline and after treatment, in order to assess functional and structural brain changes after psychotherapy. Healthy volunteers $(N=30)$ will be scanned once for comparison of baseline data. Image acquisition will be performed on a 3 Tesla scanner with a 64 Channels RF HEAD COIL (Skyra Siemens, Erlangen, Germany) at the Unit of Neuroradiology - Spedali Civili Hospital (Brescia, Italy). In order to assess the cerebral patterns of activation in response to emotional visual stimuli, during fMRI scans (EPI sequence TR/TE 2000/30 ms, voxel size of $2.2 \times 2.2 \times 3.5 \mathrm{~mm}$ ), participants will view unpleasant, 
neutral, and pleasant photographic pictures from the IAPS [63]. In particular, the fMRI study paradigm will be adapted from other studies [27, 64]. Briefly, a total of 96 intermixed unpleasant, neutral, and pleasant photographic images will be presented twice in a random order for a total of 192 trials [27, 64]. Participants will be instructed to watch the picture and then make a three-choice response (unpleasant, neutral, and pleasant) with their dominant hand, basing on the meaning for them personally. Lastly, in order to check that participants have properly understood the task, they will view the same 96 pictures immediately following the scan and will evaluated them using the Self-Assessment Manikin scale (9-point scale) [65].

\section{Sample size}

We consider as primary outcome the DERS score and assume quite homogenous population in terms of DERS scores among the recruitment centers. Previous studies reported that the standard deviation (SD) of DERS was 20.7 in females and 18.8 in males [36]. Thus, we assumed a SD of about 20 points. In addition, the correlation between two evaluations (12 months apart) was expected around 0.7 (indicating that about $50 \%$ of the variance of the second measurement should be explained by the first measurement). We computed that the SD of DERS changes will be about 15 . Our hypothesis is that MIT will decrease DERS more than SCM and, more precisely, a difference between MIT and SCM mean effect larger than 10 points will be considered clinically relevant. To recognize such difference as statistically significant (at bilateral alpha level of 0.05 ) with an adequate power $(0.80)$, the total number of patients to be recruited should be 60 , raised up to 80 (40 MIT $+40 \mathrm{SCM}$ ), considering an attrition rate around $20 \%$.

\section{Randomization}

After baseline assessments, eligible participants will be assigned to treatment arms using randomly generated block randomization scheme within each center. Considering the trade-off between the clinical best practices (e.g. The minimization of waiting list for patients) and rigorous statistical-methodological procedures, block size is fixed equal to 4 . With this block size and the two arms (named 0,1), there are 6 different ways -type of blocks Bi- (as result of combination of 4 subjects into 2-element grouping) to allocate the patients: B1 [0011]; B2 [0101]; B3 [0110]; B4 [1010]; B5 [1001]; B6 [1100].

The 80 patients of the project will be randomly assigned to the two arms by a random choice (with replacing) of 20 blocks among the six blocks above defined. For the random choice, the 'sample' command of the statistical software R was used (sample $(1: 6,20$, replace $=T)$ ). Random allocation will be done by a statistician. Within 2 weeks, the clinician in charge for clinical evaluation will enrolled the patients and will communicate the experimental arms.

Given the nature of the psychological treatment neither the therapists nor the participants can be blinded for the delivered treatment.

\section{Data management and storage}

Data will be manually entered into a database. Data will be stored at the study site following all the secure procedures: demographical and clinical information will be kept locked in dedicated spaces with limited public access. After obtaining informed consent, each participants will be associated with an alphanumeric unique code. Both the database including demographical and clinical information in an anonymous way and the file containing the name of the participants and their codes will be stored on a secure server and they will be protected by passwords. Only authorized research personnel will be access to the database.

\section{Statistical analyses}

An evaluation of type of missing data will be performed in order to detect any missing not-at-random outcome data. A subsequent data-imputation technique (Bayesian imputation) will be applied to obtain complete outcome data. Descriptive statistics will be carried out with parametric and non-parametric tests accordingly to probability data distribution. The analyses of correlation between metacognitive profiles and specific clinical and morphological brain features (i.e. cortical thickness, regional volumes) will be assessed by linear and/or generalized linear models. For longitudinal analyses we will adopt the generalized linear mixed model or generalized estimating equation (GEE) models based on the covariance structure of the data. Lastly, to identify possible predictors of response, we will adopt logistic regression models where clinical and brain markers will be the covariates and the treatment response will be the dichotomous outcome.

\section{Neuroimaging analyses}

Neuroimaging analyses will aim to 1 . assess the structural and functional correlates of BPD, 2. identify biological markers as possible predictors of treatment response, and 3 . assess the variations after psychotherapy in cortical and subcortical functional activation in response to a standardized emotional material [63] (Lang et al. 2007) along with structural and functional connectivity. Structural measures will be assessed on MRI using both whole brain cortical thickness analyisis and Region of interest (ROI) analysis of the key subregions involved in emotion regulation (e.g, amygdala). Functional measures will be assessed on task fMRI through a voxel-wise and ROI-Analyses analysis of the fluctuations in the BOLD signal. Structural 
connectivity analysis will include the assessment of fractional anisotropy, mean, axial and radial diffusivity in the major white matter tracts. The analysis will be carried out both with voxel-wise analysis and ROI approach restricted to the tracts involved in emotion (e.g., limbic tracts).

\section{Dissemination}

Results of the study will be presented at international scientific congresses and published in international scientific journals.

\section{Discussion}

The relevance of the project is twofold. First, the population included in the project represents a clinical priority of mental health system for several reasons (high suicidal risk, high direct and indirect costs, long-term impairment and social dysfunctioning), moreover in Italy there is a paucity of intervention specifically oriented to this clinical group.

Secondly, our project's contribution will be to test the effectiveness of a psychotherapeutic approach and to identify the clinical and neurobiological factors associated with response to the treatment. The inclusion of a multidisciplinary study protocol will allow to study BPD considering different features that can affect the treatment response and their reciprocal relationships.

The RCT CLIMAMITHE will contribute to deepen this topic, studying in particular the change in emotion dysregulation comparing the two treatments and the relation between these and changes in neurobiological aspects. The rationale to include emotional dysregulation as primary outcome is manifold. From a clinical point of view, emotional dysregulation is one of the core dimensions of BPD and the relationship between emotion regulation capacities and metacognitive abilities has not been yet clearly addressed. In the definition proposed by Gratz and Roemer [36], emotion regulation includes also metacognitive aspects (awareness and understanding of emotions, the ability to control impulsive behaviors and to behave flexibly in accordance with desired goals when experiencing negative emotions). We will investigated the relationship between emotion regulation, metacognitive abilities and other BPD features. From a methodological point of view, DERS has demonstrated good psychometric properties, in terms of internal consistency, test-retest reliability and validity $[36,37]$. Lastly, DERS has been previously used to measure clinical and neurobiological change after DBT [27].

The use of the same behavioral task during fMRI used in other study will let the results to be comparable and this could diminish the variabilities of their interpretation. Moreover, the expected number of patients to be included could overcome the limitations of other studies with a small sample.
In addition to ED, another core feature of BPD is the deficit in mentalizing, but no RCT with neuroimaging focused on this variables. By investigating specifically metacognition, this study will permit to contribute to the study on the mechanism of change in BPD treatments and help to provide date to the analisys on "what is expected to change in BPD?" and "which are the specific aspects that mainly contribute to the improvement of the patients' symptomatology". In fact also changes in other clinical variables will be investigate and correlate to neuroimaging data.

Moreover, BPD is a heterogeneous diagnosis with numerous comorbidities and different clinical endophenotypes based on subgroups of symptoms (impulsive symptoms, cognitive symptoms, interpersonal symptoms and affective symptoms) [66, 67]. We plan to search for specific pattern in the sample and investigate the relationship with the neurobiological variables.

This is the first RCT on BPD conducted in Italy.

\section{Strengths and limitations}

The main strength of the project is the synergy between clinical and biological expertise. Although psychotherapies are delivered in routine clinical settings, a dedicated research team coordinates and supports the activities of the project. The periodic supervision and the systematic assessment of fidelity delivered by the authors of the MIT is an effort to reduce variability and to maximize the treatment adherence. The multidimensional clinical assessment gives the opportunity to characterize BPD patients.

One possible limitation is represented by the presence (possible but not mandatory) of pharmacotherapy, which could have an impact both on clinical and neurobiological aspects. Although the pharmacotherapy does not represent the first choice in the treatment of BPD patients, it is well documented that pharmacotherapy is very common both in the United States and in Europe, with a percentage of patients prescribed medication ranging between $70 \%$ to more than $80 \%$ [68-70]. Furthermore, polypharmacy is also a common practice, with more than one-third of participants with BPD in these studies taking at least three drugs. One possible strategy to avoid that the presence of medication represents a potential confounding factor on clinical and neurobiological outcomes could be the enrollment of drug-naïve/ free patients. On the one hand, this strategy could be potentially useful, on the other hand it would lead to include a group of patients that might not be representative of the clinical real world where BPD patients are commonly treated with pharmacotherapy. We will minimize this source of variability by using a standard methodology to prescribe pharmacotherapy [1]. Furthermore, the large number of subjects will allow us to conduct additional analyses on sub-groups of patients 
who were given similar treatments. Another critical aspect could be adherence to the psychotherapy, but in the definition of the sample size we considered an attrition rate of $20 \%$ to mitigate this problem, coherently with other similar study.

\section{Trial status}

The protocol was registered on ClinicalTrials.gov with the number identifier of NCT02370316. The recruitment started 22/5/2015 and the end of the study is expected on November 2018.

\section{Additional file}

Additional file 1: SPIRIT 2013 Checklist. (DOC 128 kb)

\section{Abbreviations}

DBT: Dialectical Behavioural Therapy; DERS: Difficulties in Emotion Regulation Scale; MAl: Metacognitive Assessment Interview; MIT: Metacognitive Interpersonal Therapy; SCM: Structured Clinical Managment

\footnotetext{
Acknowledgements Not applicable.

*CLIMAMITHE Study group: Roberta Rossi (IRCCS Istituto Centro San Giovanni di Dio Fatebenefratelli, Brescia), Laura Rosa Magni (IRCCS Istituto Centro San Giovanni di Dio Fatebenefratelli, Brescia), Mariangela Lanfredi (IRCCS Istituto Centro San Giovanni di Dio Fatebenefratelli, Brescia), Laura Pedrini (IRCCS Istituto Centro San Giovanni di Dio Fatebenefratelli, Brescia), Antonino Carcione (Third Centre of Cognitive Psychotherapy, Rome), Antonio Semerari (Third Centre of Cognitive Psychotherapy, Rome), Ilaria Riccardi (Third Centre of Cognitive Psychotherapy, Rome), Giuseppe Nicolo' (Third Center of Cognitive Psychotherapy and Dipartimento Salute Mentale Roma 5, Rome), Monica Almici (IRCCS Istituto Centro San Giovanni di Dio Fatebenefratelli, Brescia), Rossella Beneduce (IRCCS Istituto Centro San Giovanni di Dio Fatebenefratelli, Brescia), Genoveffa Borsci (IRCCS Istituto Centro San Giovanni di Dio Fatebenefratelli, Brescia), Chiara Caprioli (IRCCS Istituto Centro San Giovanni di Dio Fatebenefratelli, Brescia), Matteo Nodari (IRCCS Istituto Centro San Giovanni di Dio Fatebenefratelli, Brescia), Antonio Vita (Department of Mental Health, ASST Spedali Civili of Brescia), Stefano Barlati (Department of Mental Health, ASST Spedali Civili of Brescia), Laura Laffranchini (IRCCS Istituto Centro San Giovanni di Dio Fatebenefratelli, Brescia), Luciana Rillosi (IRCCS Istituto Centro San Giovanni di Dio Fatebenefratelli, Brescia), Giuseppe Rossi (IRCCS Istituto Centro San Giovanni di Dio Fatebenefratelli, Brescia), Luisella Bocchio (IRCCS Istituto Centro San Giovanni di Dio Fatebenefratelli, Brescia), Anna Cattaneo (IRCCS Istituto Centro San Giovanni di Dio Fatebenefratelli, Brescia), Nadia Cattane (IRCCS Istituto Centro San Giovanni di Dio Fatebenefratelli, Brescia), Giovanni Battista Tura (IRCCS Istituto Centro San Giovanni di Dio Fatebenefratelli, Brescia), Stefano Bignotti (IRCCS Istituto Centro San Giovanni di Dio Fatebenefratelli, Brescia), Maddalena Speziali (IRCCS Istituto Centro San Giovanni di Dio Fatebenefratelli, Brescia), Maria Cotelli (IRCCS Istituto Centro San Giovanni di Dio Fatebenefratelli, Brescia), Sandra Rosini (IRCCS Istituto Centro San Giovanni di Dio Fatebenefratelli, Brescia), Roberto Gasparotti (ASST Spedali Civili, Università degli Studi di Brescia), Claudia Ambrosi (ASST Spedali Civili, Brescia), Lorella Mascaro (ASST Spedali Civili, Brescia), Daniele Corbo (ASST Spedali Civili, Brescia), Michela Pievani (IRCCS Istituto Centro San Giovanni di Dio Fatebenefratelli, Brescia), Giulia Quattrini (IRCCS Istituto Centro San Giovanni di Dio Fatebenefratelli, Brescia), Elena Bilotta (Third Centre of Cognitive Psychotherapy, Rome), Livia Colle (Third Centre of Cognitive Psychotherapy, Rome), Laura Conti (Third Centre of Cognitive Psychotherapy, Rome), Donatella Fiore (Third Centre of Cognitive Psychotherapy, Rome), Alessandra Micheloni (Third Centre of Cognitive Psychotherapy, Rome), Michele Procacci (Third Centre of Cognitive Psychotherapy, Rome), Valentina Silvestre (Third Centre of Cognitive Psychotherapy, Rome).
}

\section{Funding}

This trial was funded by a National funding by Italian Ministry of Health in the framework of the grant "BANDO 2011-2012 GIOVANI RICERCATORI e RICERCA FINALIZZATA"(GR-2011-02351347).

\section{Availability of data and materials Not applicable.}

\section{Authors' contributions}

$L R M, A C, A S, I R, G N, M L, L P, M P, R G, R R$ and all the members of the CLIMAMITHE Group contribute to the data collection and will participate actively to the treatments. LRM, RR drafted and critically revised the manuscript, $A C, C F, A S, G N M L, L P, M P, M C$, LB, RG, critically revised the manuscript. All authors read and approved the final manuscript.

\section{Ethics approval and consent to participate}

There are no immediate ethical problems regarding this trial. Research has not identified any significant adverse effects or risks from any of the compared interventions, and we have no advance knowledge of which intervention is most efficacious. During the trial period, any adverse event will be reported.

The trial is approved by the Comitato Etico IRCCS Fatebenefratelli (number 67/2014), and is registered under Clinical Trials.org as NCT02370316. The participation in the study is voluntary. All participants signed a written informed consent approved by the Comitato Etico IRCCS Fatebenefratelli after that a staff member has explained in detail the aim and the design of the study. Participants are informed that if decide not to participate in the study their decision will not affect their treatment in any way. Lastly, they are informed that the can withdraw their participation at any time. In accordance with the SPIRIT guidelines [37], we will report positive, negative, and neutral findings in the trial and we describe the RCT in accordance to the SPIRIT checklist and flow-chart for Randomized Controlled Trials of nonpharmacologic treatments [37] (please see Additional file 1).

\section{Consent for publication}

Not applicable.

\section{Competing interests}

The authors declare that they have no competing interests.

\section{Publisher's Note}

Springer Nature remains neutral with regard to jurisdictional claims in published maps and institutional affiliations.

\section{Author details \\ ${ }^{1}$ Unit of Psychiatry, IRCCS Istituto Centro San Giovanni di Dio Fatebenefratelli, Brescia, Italy. ${ }^{2}$ Third Centre of Cognitive Psychotherapy, Rome, Italy. ${ }^{3}$ Italian School of Cognitive Psychotherapy (SICC), Rome, Italy. ${ }^{4}$ Service of Statistics, IRCCS Istituto Centro San Giovanni di Dio Fatebenefratelli, Brescia, Italy. ${ }^{5}$ Dipartimento Salute Mentale Roma 5, Rome, Italy. ${ }^{6}$ Neuropsychology Unit, IRCCS Istituto Centro San Giovanni di Dio Fatebenefratelli, Brescia, Italy. ${ }^{7}$ IRCCS Istituto Centro S. Giovanni di Dio, Fatebenefratelli, Brescia, Italy. ${ }^{8}$ Laboratory Alzheimer's Neuroimaging and Epidemiology, IRCCS Istituto Centro San Giovanni di Dio Fatebenefratelli, Brescia, Italy. ${ }^{9}$ Neuroradiology Unit, Department of Medical and Surgical Specialties, Radiological Sciences, and Public Health, University of Brescia, Brescia, Italy.}

Received: 31 October 2018 Accepted: 26 April 2019

Published online: 24 June 2019

References

1. APA, American Psychiatric Association. Practice guideline for the treatment of patients with borderline personality disorder. Am J Psychiatry. 2001;158: $1-52$.

2. McMain SF, Links PS, Gnam WH, Guimond T, Cardish RJ, Korman L, Streiner $\mathrm{DL}$. A randomized trial of dialectical behavior therapy versus general psychiatric management for borderline personality disorder. Am J Psychiatry. 2009;166(12):1365-74.

3. Bateman AW, Fonagy P. Mentalization-based treatment of BPD. J Personal Disord. 2004;18(1):36-51. 
4. Clarkin JF, Levy KN, Lenzenweger MF, Kernberg OF. Evaluating three treatments for borderline personality disorder: a multiwave study. Am J Psychiatry. 2007;164(6):922-8.

5. Holmes EA, Craske MG, Graybiel AM. sychological treatments: a call for mental-health science. Nature. 2014;511(7509):287-9.

6. Leichsenring F, Leibing E, Kruse J, New AS, Leweke F. Borderline personality disorder. Lancet. 2011;377(9759):74-84.

7. Nunes PM, Wenzel A, Borges KT, Porto CR, Caminha RM, de Oliveira IR. Volumes of the hippocampus and amygdala in patients with borderline personality disorder: a meta-analysis. J Personal Disord. 2009;23(4):333-45.

8. Goodman M, Hazlett EA, Avedon JB, Siever DR, Chu KW, New AS. Anterior cingulate volume reduction in adolescents with borderline personality disorder and co-morbid major depression. J Psychiatr Res. 2011;45(6):803-7.

9. Rossi R, Lanfredi M, Pievani M, Boccardi M, Beneduce R, Rillosi L, Giannakopoulos P. Thompson PM, Rossi G, Frisoni GB. Volumetric and topographic differences in hippocampal subdivisions in borderline personality and bipolar disorders. Psychiatry Res. 2012;203(2-3):132-8.

10. Rossi R, Pievani M, Lorenzi M, Boccardi M, Beneduce R, Bignotti S, Borsci G, Cotelli M, Giannakopoulos P, Magni LR, Rillosi L, Rosini S, Rossi G, Frisoni GB. Structural brain features of borderline personality and bipolar disorders. Volumetric and topographic differences in hippocampal subdivisions in borderline personality and bipolar disorders. Psychiatry Res. 2013;213(2):83-91.

11. Sala M, Caverzasi E, Lazzaretti M, Morandotti N, De Vidovich G, Marraffini E, d'Allio G. Dorsolateral prefrontal cortex and hippocampus sustain impulsivity and aggressiveness in borderline personality disorder. J Affect Disord. 2011;131(1-3):417-21.

12. Soloff $P$, Pruitt $P$, Sharma M, Radwan J, White R, Diwadkar VA. Structural brain abnormalities and suicidal behavior in borderline personality disorder. J Psychiatr Res. 2012;46(4):516-25.

13. Rossi R, Lanfredi M, Pievani M, Boccardi M, Rasser PE, Thompson PM, Cavedo E, Cotelli M, Rosini S, Beneduce R, Bignotti S, Magni LR, Rillosi L, Magnaldi S, Cobelli M, Rossi G, Frisoni GB. Abnormalities in cortical gray matter density in borderline personality disorder. Eur Psychiatry. 2015;30(2): $221-7$.

14. Soloff P, Nutche J, Goradia D, Diwadkar V. Structural brain abnormalities in borderline personality disorder: a voxel-based morphometry study. Psychiatry Res. 2008;164(3):223-36.

15. Mauchnik J, Schmahl C. The latest neuroimaging findings in borderline personality disorder. Curr Psychiatry Rep. 2010;12(1):46-55.

16. Saxena S, Rauch SL. Functional neuroimaging and the neuroanatomy of obsessive-compulsive disorder. Psychiatr Clin North Am. 2000;23(3):563-86.

17. Nakatani E, Nakgawa A, Ohara Y, Goto S, Uozumi N, Iwakiri M, Yamagami T. Effects of behavior therapy on regional cerebral blood flow in obsessivecompulsive disorder. Psychiatry Res Neuroimaging. 2003;124(2):113-20.

18. Schwartz JM, Stoessel PW, Baxter LR Jr, Martin KM, Phelps ME. Systematic changes in cerebral glucose metabolic rate after successful behavior modification treatment of obsessive-compulsive disorder. Arch Gen Psychiatry. 1996;53(2):109-13.

19. Brody AL, Saxena S, Stoessel P, Gillies LA, Fairbanks LA, Alborzian S, Phelps ME, Huang SC, Wu HM, Ho ML, Ho MK, Au SC, Maidment K, Baxter LR Jr. Regional brain metabolic changes in patients with major depression treated with either paroxetine or interpersonal therapy: preliminary findings. Arch Gen Psychiatry. 2001:58(7):631-40.

20. Goldapple K, Segal Z, Garson C, Lau M, Bieling P, Kennedy S, Mayberg H. Modulation of cortical-limbic pathways in major depression: treatmentspecific effects of cognitive behavior therapy. Arch Gen Psychiatry. 2004 61(1):34-41.

21. Martin SD, Martin E, Rai SS, Richardson MA, Royall R. Brain blood flow changes in depressed patients treated with interpersonal psychotherapy or venlafaxine hydrochloride: preliminary findings. Arch Gen Psychiatry. 2001; 58(7):641-8

22. Prasko J, Horácek J, Záleský R, Kopecek M, Novák T, Pasková B, Skrdlantová $\mathrm{L}$, Belohlávek O, Höschl C. The change of regional brain metabolism (18FDG PET) in panic disorder during the treatment with cognitive behavioral therapy or antidepressants. Neuro Endocrinol Lett. 2004;25(5):340-8.

23. Furmark T, Tillfors $M$, Marteinsdottir I, Fischer $H$, Pissiota $A$, Långström $B$, Fredrikson M. Common changes in cerebral blood flow in patients with social phobia treated with citalopram or cognitive-behavioral therapy. Arch Gen Psychiatry. 2002;59(5):425-33.

24. Paquette $V$, Lévesque J, Mensour B, Leroux JM, Beaudoin $G$, Bourgouin $P$, Beauregard M. "Change the mind and you change the brain": effects of cognitive-behavioral therapy on the neural correlates of spider phobia. Neuroimage. 2003;18(2):401-9.

25. Straube T, Glauer M, Dilger S, Mentzel HJ, Miltner WH. Effects of cognitivebehavioral therapy on brain activation in specific phobia. Neuroimage. 2006; 29(1):125-35.

26. Felmingham K, Kemp A, Williams L, Das P, Hughes G, Peduto A, Bryant R. Changes in anterior cingulate and amygdala after cognitive behavior therapy of posttraumatic stress disorder. Psychol Sci. 2007;18(2):127-9.

27. Goodman M, Carpenter D, Tang CY, Goldstein KE, Avedon J, Fernandez N, Mascitelli KA, Blair NJ, New AS, Triebwasser J, Siever LJ, Hazlett EA. Dialectical behavior therapy alters emotion regulation and amygdala activity in patients with borderline personality disorder. J Psychiatr Res. 2014;57: $108-16$.

28. Schnell K, Herpertz SC. Effects of dialectic-behavioral-therapy on the neural correlates of affective hyperarousal in borderline personality disorder. J Psychiatr Res. 2007;41(10):837-47

29. Schmitt R, Winter D, Niedtfeld I, Herpertz SC, Schmahl C. Effects of Psychotherapy on Neuronal Correlates of Reappraisal in Female Patients With Borderline Personality Disorder. Biol Psychiatry Cogn Neurosci Neuroimaging. 2016;1(6):548-57.

30. Winter D, Niedtfeld I, Schmitt R, Bohus M, Schmahl C, Herpertz SC. Neural correlates of distraction in borderline personality disorder before and after dialectical behavior therapy. Eur Arch Psychiatry Clin Neurosci. 2017;267(1): 51-62.

31. Mancke F, Schmitt R, Winter D, Niedtfeld I, Herpertz SC, Schmahl C. Assessing the marks of change: how psychotherapy alters the brain structure in women with borderline personality disorder. J Psychiatry Neurosci. 2017:43(1):170132.

32. Perez DL, Vago DR, Pan H, Root J, Tuescher O, Fuchs BH, Leung L, Epstein J, Cain NM, Clarkin JF, Lenzenweger MF, Kernberg OF, Levy KN, Silbersweig DA, Stern E. Frontolimbic neural circuit changes in emotional processing and inhibitory control associated with clinical improvement following transference-focused psychotherapy in borderline personality disorder. Psychiatry Clin Neurosci. 2016;70(1):51-61.

33. Semerari A, Carcione A, Dimaggio G, Falcone M, Nicolò G, Procacci M, Alleva G, Mergenthaler E. Assessing problematic states in patients' narratives: the grid of problematic states. Psychother Res. 2003;13(3):337-53.

34. Carcione A, Nicolò G, Pedone R, Popolo R, Conti L, Fiore D, Procacci M, Semerari A, Dimaggio G. Metacognitive mastery dysfunctions in personality disorder psychotherapy. Psychiatry Res. 2011;190(1):60-71.

35. Carcione A, Nicolò G, Semerari A. Curare i casi complessi. La terapia metacognitiva interpersonale dei disturbi di personalità. Editore Laterza, 2017.

36. Gratz $\mathrm{J}$, Roemer L. Multidimensional assessment of emotion regulation and dysregulation: development, factor structure, and initial validation of the difficulties in emotion regulation scale. J Psychopathol Behav Assess. 2004; 26:1.

37. Giromini L, Velotti P, de Campora G, Bonalume L, Cesare ZG. Cultural adaptation of the difficulties in emotion regulation scale: reliability and validity of an Italian version. J Clin Psychol. 2012;68(9):989-1007.

38. Chan AW, Tetzlaff JM, Gøtzsche PC, Altman DG, Mann H, Berlin JA, Dickersin K, Hróbjartsson A, Schulz KF, Parulekar WR, Krleza-Jeric K, Laupacis A, Moher D. SPIRIT 2013 explanation and elaboration: guidance for protocols of clinical trials. BMJ. 2013;346:e7586.

39. First MB, Spitzer RL, Gibbon M, Williams JBW. Structured clinical interview for DSM-IV Axis I disorders - patient edition (SCID-I/P). New York: New York State Psychiatric Institute, Biometric Research Department; 1995.

40. First MB, Spitzer RL, Gibbon M, Williams JBW, Benjamin L. Structured clinical interview for DSM-IV Axis II personality disorders (SCID-II). New York: New York State Psychiatric Institute, Biometric Research Department; 1994.

41. Dimaggio G, Carcione A, Salvatore G, Semerari A, Nicolò G. A rational model for maximizing the effects of therapeutic relationship regulation in personality disorders with poor metacognition and over-regulation of affects. Psychol Psychother. 2010;83(4):363-84

42. Dimaggio G, Semerari A. Disturbi di personalità. Modelli e trattamento. Stati mentali, metarappresentazione, cicli interpersonali, Editore Laterza; 2007.

43. Carcione A, Dimaggio G, Fiore D, Nicolò G, Procacci M, Semerari A, Pedone R. An intensive case analysis of client metacognition in a good-outcome psychotherapy: Lisa's case. Psychother Res. 2008;18(6):667-76.

44. Semerari A, Cucchi M, Dimaggio G, Cavadini D, Carcione A, Battelli V, Nicolò G, Pedone R, Siccardi T, D'Angerio S, Ronchi P, Maffei C, Smeraldi E. The 
development of the Metacognition Assessment interview: instrument description, factor structure and reliability in a non-clinical sample. Psychiatry Res. 2012;200(2-3):890-5.

45. Bateman, Krawitz. Borderline Personality Disorder: an evidence-based guide for generalist mental health professionals Oxford. New York: Oxford University Press; 2013.

46. Bateman A, Fonagy P. Randomized controlled trial of outpatient mentalization-based treatment versus structured clinical management for borderline personality disorder. Am J Psychiatry. 2009;166(12):1355-64.

47. Pellecchia G, Moroni F, Carcione A, Colle L, Dimaggio G, Nicolò G, et al. Metacognition assessment interview: instrument description and factor structure. Clin Neuropsychiatry. 2015;12:156-67.

48. Bilotta E, Carcione A, Fera T, Moroni F, Nicolò G, Pedone R, Pellecchia G Semerari A, Colle L. Symptom severity and mindreading in narcissistic personality disorder. PLoS One. 2018;13(8):e0201216.

49. Derogatis LR. Symptom Checklist-90-R: administrative scoring and procedures manual. Minneapolis: NCS Pearson; 1994.

50. Beck AT, Steer RA, Brown GK. Manual for the Beck depression inventory-ll. San Antonio: Psychological Corporation; 1996.

51. Spielberger CD. The state-trait anger expression Inventory-2 (STAXI-2): professional manual. Odessa: Psychological Assessment Resources, Inc; 1999.

52. Patton JH, Stanford MS, Barratt ES. Factor structure of the Barratt impulsiveness scale. J Clin Psychol. 1995;51(6):768-74.

53. Pilkonis PA, Kim Y, Proietti JM. Scales for personality disorders developed from the inventory of interpersonal problems. J Personal Disord. 1996;10: 355-69.

54. Bagby RM, Parker JD, Taylor GJ. The twenty-item Toronto Alexithymia Scale--I. Item selection and cross-validation of the factor structure. J Psychosom Res. 1994;38(1):23-32.

55. Bernstein DP, Fink L. Childhood trauma questionnaire: a retrospective selfreport manual. San Antonio: The Psychological Corporation; 1998.

56. Feeney JA, Noller P, Hanrahan, M. Assessing adult attachment in: Sperling, M. B. Berman W. H. Attachment in adults: Clinical and developmental perspectives. New York: Guilford Press, 1994. p. 128-152.

57. Lezak MD, Howieson DB, Loring DW, Fischer JS. Neuropsychological assessment. USA: Oxford University Press; 2004.

58. Davis MH. Measuring individual differences in empathy: evidence for a multidimensional approach. J Pers Soc Psychol. 1993;44:113-26.

59. Baron-Cohen S, Wheelwright S, Hill J, Raste Y, Plumb I. The "Reading the mind in the eyes" test revised version: a study with normal adults, and adults with Asperger syndrome or high-functioning autism. J Child Psychol Psychiatry. 2001:42:241-51.

60. Kessler H, Bayerl P, Deighton RM, Traue HC. Facially expressed emotion labeling (FEEL): PC-gestützter test zur Emotionserkennung. Verhaltenstherapie und Verhaltensmedizin. 2002;23(3):297-306.

61. Manenti R, Brambilla M, Cotelli M. Age-related changes in implicit emotion processing. Aging Neuropsychol Cognit. 2019;26(1):86-104 Epub 2017 Nov 28.

62. Galluzzi S, Marizzoni M, Babiloni C, Albani D, Antelmi L, Bagnoli C, BartresFaz D, Cordone S, Didic M, Farotti L, Fiedler U, Forloni G, Girtler N, Hensch T, Jovicich J, Leeuwis A, Marra C, Molinuevo JL, Nobili F, Pariente J, Parnetti L, Payoux P, Del Percio C, Ranjeva JP, Rolandi, Rossini PM, Schönknecht P, Soricelli A, Tsolaki M, Visser PJ, Wiltfang J, Richardson JC, Bordet R, Blin O, Frisoni GB, PharmaCog Consortium. Clinical and biomarker profiling of prodromal Alzheimer's disease in workpackage 5 of the Innovative Medicines Initiative PharmaCog project: a 'European ADNI study'. J Intern Med. 2016;279(6):576-91.

63. Lang P, Bradley MM. The international affective picture system (IAPS) in the study of emotion and attention. In: Handbook of emotion elicitation and assessment, 29; 2007.

64. Hazlett EA, Zhang J, New AS, Zelmanova Y, Goldstein KE, Haznedar MM, Meyerson D, Goodman M, Siever L, Chu KW. Potentiated amygdala response to repeated emotional pictures in borderline personality disorder. Biol Psychiatry. 2012;72(6):448-56.

65. Bradley MM, Lang PJ. Measuring emotion: the self-assessment manikin and the semantic differential. J Behav Ther Exp Psychiatry. 1994;25(1):49-59.

66. Gunderson JG, Stout RL, McGlashan TH, Shea MT, Morey LC, Grilo CM, Zanarini MC, Yen S, Markowitz JC, Sanislow C, Ansell E, Pinto A, Skodol AE. Ten-year course of borderline personality disorder: psychopathology and function from the collaborative longitudinal personality disorders study. Arch Gen Psychiatry. 2011;68(8):827-37.
67. Paris J. The diagnosis of borderline personality disorder: problematic but better than the alternatives. Ann Clin Psychiatry. 2005;17(1):41-6.

68. Zanarini MC, Frankenburg FR, Bradford Reich D, Harned AL, Fitzmaurice GM. Rates of psychotropic medication use reported by borderline patients and axis II comparison subjects over 16 years of prospective follow-up. I Clin Psychopharmacol. 2015;35(1):63-7.

69. Bridler R, Häberle A, Müller ST, Cattapan K, Grohmann R, Toto S, Greil W. Psychopharmacological treatment of 2195 in-patients with borderline personality disorder: a comparison with other psychiatric disorders. Eur Neuropsychopharmacol. 2015;25(6):763-72.

70. Martín-Blanco A, Ancochea A, Soler J, Elices M, Carmona C, Pascual JC. Changes over the last 15 years in the psychopharmacological management of persons with borderline personality disorder. Acta Psychiatr Scand. 2017; 136(3):323-31.
Ready to submit your research? Choose BMC and benefit from:

- fast, convenient online submission

- thorough peer review by experienced researchers in your field

- rapid publication on acceptance

- support for research data, including large and complex data types

- gold Open Access which fosters wider collaboration and increased citations

- maximum visibility for your research: over $100 \mathrm{M}$ website views per year

At BMC, research is always in progress.

Learn more biomedcentral.com/submissions 